\title{
Optical Properties of BN and BBi Compounds
}

\author{
B.G. Yalcin*, M. Ustundag and M. Aslan \\ Sakarya University, Art\&Science Faculty, Department of Physics \\ Esentepe Campus, 54187, Serdivan, Sakarya, Turkey
}

\begin{abstract}
We have investigated optical properties which are very important for optoelectronic devices, such as laser modulators, photo detectors, optical amplifier and high efficient solar cells of BN and BBi using the density functional theory based on full potential linearized augmented plane wave method as implemented in Vienna ab-initio simulation package (VASP). The exchange correlation potential is treated by generalized gradient approximation. Primarily, we have calculated the equilibrium lattice constant $\left(a_{0}\right)$ of BN and BBi. Our results for $a_{0}$ are $3.6264 \AA$ and $5.5243 \AA$ for BN and BBi, respectively. These results fairly coincide with theoretical and experimental studies. Then, we have calculated the optical parameters (dielectric functions, absorption and refractive index, reflectivity, energy loss function and conductivity) of $\mathrm{BN}$ and $\mathrm{BBi}$ which is directly related to the energy band structure of the material.
\end{abstract}

DOI: $10.12693 /$ APhysPolA.125.574

PACS: 71.55.Eq, 71.15.Mb, 78.20.Ci

\section{Introduction}

Boron-V compounds have great technological importance for electronic and optoelectronic applications due to their some extreme physical properties such as hardness, low ionicity, and high thermal conductivity [1]. For this reason, the structural and electronic properties of $\mathrm{B}-\mathrm{V}$ compounds have been studied both theoretically and experimentally.

In this work, we present optical and electronic properties of $\mathrm{BN}$ and $\mathrm{BBi}$, the first and the last members of $\mathrm{B}-\mathrm{V}$ compounds. The optical dielectric function of $\mathrm{BN}$ are studied by Satta et al. [2]. The structural and electronic properties of $\mathrm{BBi}$ is studied by Cui et al. [3] and Ferhat and Zaoui [4]. To our knowledge there is no experimental and theoretical work on the optical properties of $\mathrm{BBi}$. To understand how the strong disparity in atomic size affects the electronic and optical properties of B-V compounds, we present a comprehensive work on the electronic and optical properties of $\mathrm{BN}$ and $\mathrm{BBi}$.

\section{Method of calculation}

In this study, all our calculations are implemented by the Vienna ab-initio simulation package (VASP) [5-7] within density functional theory (DFT) $[8,9]$. The exchange-correlation energy function is treated within the generalized gradient approximation (GGA) [10]. Wave functions are expanded by the plane waves up to a cutoff kinetic energy of $400 \mathrm{eV}$. Integration over the Brillouin zone is performed by using the special $k$-point sampling of the Gamma-centered Monkhorst-Pack scheme [11] with a $12 \times 12 \times 12$ grid and the self-consistent convergence of the total energy is $10^{-6} \mathrm{eV} /$ atom. The Fermi

*corresponding author; e-mail: gyalcin@sakarya.edu.tr level is smeared by the Gaussian method with a width of $0.2 \mathrm{eV}$. The valence electronic configurations of the atoms considered here are as follows: B: $\left[2 s^{2} 2 p^{1}\right], \mathrm{N}:\left[2 s^{2} 2 p^{3}\right]$ and $\mathrm{Bi}:\left[5 d^{10} 6 s^{2} 6 p^{3}\right]$. In zinc-blende $\mathrm{BN}$ and $\mathrm{BBi}$ the $\mathrm{B}, \mathrm{N}$ and $\mathrm{Bi}$ atoms are the positions of $\mathrm{B}(0,0,0)$ and $\mathrm{N} / \mathrm{Bi}(1 / 4,1 / 4,1 / 4)$. In this study, we have calculated the equilibrium lattice constant $\left(a_{0}\right)$. The whole electronic and optical calculations are obtained by using $a_{0}$.

\section{Results and discussions}

As in the first step of our calculations, we have calculated the equilibrium lattice constant of $\mathrm{BN}$ and $\mathrm{BBi}$ compounds in stabilized zinc-blende (ZB) phase. Our obtained results are shown in Table I along with experimental and theoretical values. The lattice constant of BN is fairly in agreement with experimental [12] and theoretical [13] values within $0.3 \%$ and $0.07 \%$, respectively. It is clear that our calculated lattice constant of BBi is consistent with theoretical studies $[14,15]$. To our knowledge, there is no experimental result for the lattice constant of BBi.

TABLE I

Calculated lattice constants and band gap energies for $\mathrm{BN}$ and $\mathrm{BBi}$ compounds with the experimental data and previous theoretical calculations.

\begin{tabular}{c|c|c|c}
\hline \hline Material & $\begin{array}{c}\text { Lattice } \\
\text { constant } \\
{[\AA]}\end{array}$ & $E_{\mathrm{g}}[\mathrm{eV}]$ & Refs. \\
\hline $\mathrm{BN}$ & 3.6264 & 4.4635 (indirect) & our result \\
& 3.6150 & - & exp. [12] \\
& 3.6290 & 4.470 (indirect) & theory [13] \\
\hline $\mathrm{BBi}$ & 5.5243 & 0.1015 (direct) & our result \\
& 5.5290 & 0.130 (direct) & theory [14] \\
& 5.524 & - & theory [15]
\end{tabular}

The band structures of $\mathrm{BN}$ and $\mathrm{BBi}$ are calculated by using the equilibrium lattice constant at zero GPa. The 
electronic band structures along the various symmetry lines are calculated for both materials. As a result of our calculations, ZB structure of the BN has indirect gap, which is $4.4635 \mathrm{eV}$. This value is fairly in agreement with the theoretical work [13]. Otherwise, BBi has direct gap at $\Gamma$ symmetry point. The direct band gap energy of BBi is found as $0.1015 \mathrm{eV}$ which indicates coincidence with theoretical work [14]. We have summarized the band gap energies of $\mathrm{BN}$ and $\mathrm{BBi}$ in Table $\mathrm{I}$ with previous theoretical studies.

The optical properties of a material is generally defined according to the complex dielectric function based on frequency: $\varepsilon(\omega)=\varepsilon_{1}(\omega)+\mathrm{i} \varepsilon_{2}(\omega)$, where $\varepsilon_{1}(\omega)$ and $\varepsilon_{2}(\omega)$ are the real and imaginary parts of dielectric function, respectively. The $\varepsilon(\omega)$ can be used to describe the linear response of the system to electromagnetic radiation, which relates to the interaction of photons and electrons to obtain the optical band gap and optical transition of the $\mathrm{BN}$ and BBi compounds. The dispersion of the imaginary part $\varepsilon_{2}(\omega)$ of the dielectric function can be calculated from the momentum matrix elements between the occupied and unoccupied wave functions. The real part $\varepsilon_{1}(\omega)$ of the dielectric function can be calculated from the imaginary part $\varepsilon_{2}(\omega)$ by Kramers-Kronig relationship [16]. All of the optical constants such as reflectivity $R(\omega)$, absorption $\alpha(\omega)$, loss function $L(\omega)$, refractive index $n(\omega)$ and optical conductivity $\sigma(\omega)$ can be derived from $\varepsilon_{1}(\omega)$ and $\varepsilon_{2}(\omega)$ [17].

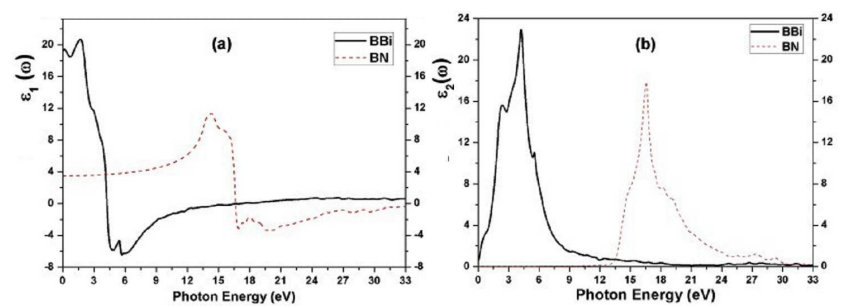

Fig. 1. Calculated (a) $\varepsilon_{1}(\omega)$ and (b) $\varepsilon_{2}(\omega)$ spectra of $\mathrm{BN}$ and $\mathrm{BBi}$.
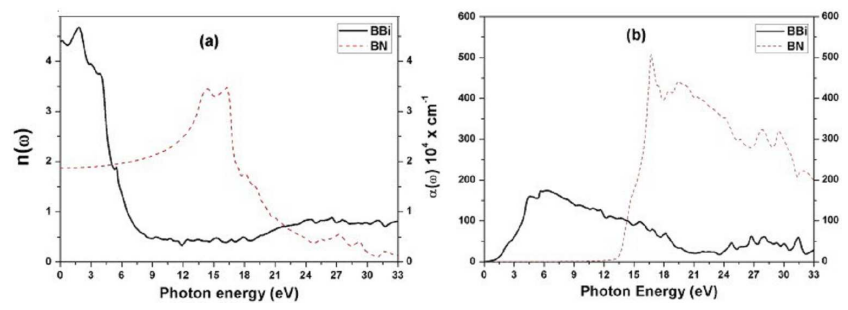

Fig. 2. (a) Refractive index spectrum and (b) absorption spectrum of $\mathrm{BN}$ and $\mathrm{BBi}$.

For $\mathrm{BN}$ and $\mathrm{BBi}$, the calculated real and imaginary parts of dielectric function are displayed in Fig. 1a and b, respectively. The main peaks of the $\varepsilon_{1}(\omega)$ are accurate as 1.7 and $14.327 \mathrm{eV}$ for $\mathrm{BBi}$ and $\mathrm{BN}$, respectively, as shown in Fig. 1a. They are generated by electron tran- sition from the top of valence band to the bottom of conduction band. An important quantity of $\varepsilon_{1}(\omega)$ is the zero frequency limit $\varepsilon_{1}(0)$, which is the electronic part of the static dielectric constant that depends strongly on the band gap. Dielectric constant $\varepsilon_{1}(0)$ of $\mathrm{BBi}$ and $\mathrm{BN}$ are found to be 19.27 and 3.5, respectively, as listed in Table II. There is a good coincidence for the zero frequency limit $\varepsilon_{1}(0)$ of $\mathrm{BN}$ with theoretical result [18]. In Fig. 1b, each $\varepsilon_{2}(\omega)$ spectrum has a prominent peak. It is located at 3.795 and $16.585 \mathrm{eV}$ for $\mathrm{BBi}$ and $\mathrm{BN}$ which corresponds to electronic passages from valance band to transmission band (optical transitions). From the dispersion curves of refractive index (Fig. 2a), the obtained static refractive index $n(0)$ values are found to be for BBi and BN 4.39 and 1.87, respectively, as listed in Table II. Our value for $n(0)$ of $\mathrm{BN}$ is in good agreement with previous results [18]. The absorption coefficient tells the decline of light intensity spreading in unit distance in medium. The absorption edge is located at around 0.2 and $6 \mathrm{eV}$ with small absorption value for $\mathrm{BBi}$ and $\mathrm{BN}$, respectively.

TABLE II

Calculated zero frequency limit $\varepsilon_{1}(\omega)$ and static reflective index $n(0)$ values for $\mathrm{BN}$ and $\mathrm{BBi}$ with other results.

\begin{tabular}{c|c|c|c}
\hline \hline Material & $\varepsilon_{1}(0)$ & $\mathrm{n}(0)$ & Refs. \\
\hline $\mathrm{BN}$ & 3.5 & 1.87 & our result \\
& 4.17 & 2.04 & theory [18] \\
$\mathrm{BBi}$ & 19.27 & 4.39 & our result
\end{tabular}

The $\alpha(\omega)$ increases rapidly, when photon energy is more than these values, which is related to optical band gap and density of states, as shown in Fig. 2 b.

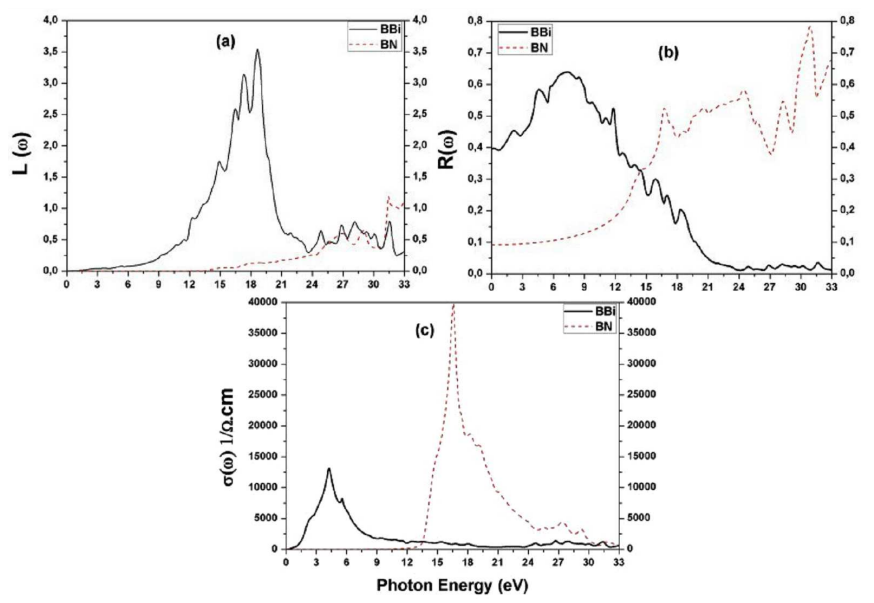

Fig. 3. (a) Energy loss spectrum, (b) reflectivity spectrum, and (c) optical conductivity of BN and BBi compounds.

$L(\omega)$ is an important constant describing the energy loss of the electrons passing between bands. In Fig. 3a, the loss function for both materials can be seen. The 
peak in the $L(\omega)$ spectra represents the plasma resonance and corresponds to the trailing edges in the $R(\omega)$ spectra. Also, the definite peaks in $L(\omega)$ are related to collective vibrations of valence electrons. The calculated optical reflectivity is shown in Fig. 3b. The calculated reflectivity is found to be 8.45 and $30.87 \mathrm{eV}$ for $\mathrm{BBi}$ and $\mathrm{BN}$, respectively. The $R(\omega)$ spectra for two compounds show several peaks which originate from interband transitions along various high symmetry points. The optical spectrum is displayed for $\mathrm{BN}$ and $\mathrm{BBi}$ in Fig. 3c. In the optical conductivity, the peak is determined by the electric-dipole transitions between the occupied and the unoccupied states.

\section{Conclusion}

In this study, we have calculated equilibrium lattice constant for BN and BBi using the VASP code. The electronic and optical properties are investigated by means of equilibrium lattice constant. Finally, the dielectric function and the dispersion properties of optical constants such as the refractive index, absorption coefficient, electron energy-loss spectrum, reflectivity, and optical conductivity are presented. The obtained results are fairly in agreement with available experimental and theoretical results.

\section{Acknowledgments}

This work is supported by Sakarya University Research Project Unit under project No. 2012-02-02-008.

\section{References}

[1] M.P. Surh, S.G. Louie, M.L. Cohen, Phys. Rev. B 43, 9126 (1991).

[2] G. Satta, G. Cappelini, M. Palummo, G. Onida, Comput. Mater. Sci. 22, 78 (2001).

[3] S. Cui, W. Feng, H. Hu, Z. Feng, Y. Wag, Comput. Mater. Sci. 47, 968 (2010).
[4] M. Ferhat, A. Zaoui, Phys. Rev. B 73, 115107 (2006).

[5] G. Kresse, J. Forthmuller, Phys. Rev. B 54, 11169 (1996).

[6] MedeA-VASP (Materials Design Inc.) is a commercial pack that provides a graphical interface to set up, run, and analyze VASP calculations under the Windows environment.

[7] G. Kresse, J. Forthmuller, Comput. Mater. Sci. 6, 15 (1996).

[8] P. Hohenberg, W. Kohn, Phys. Rev. 136, B864 (1964).

[9] W. Khon, L.S. Sham, Phys. Rev. 140, A1133 (1965).

[10] J.P. Perdew, K. Burke, M. Ernzerhof, Phys. Rev. Lett. 77, 3865 (1996).

[11] H.J. Monkhorst, J.D. Pack, Phys. Rev. B 13, 5188 (1976).

[12] R.H. Wentorf, J. Chem. Phys. 26, 956 (1957).

[13] R. Ahmed, F. Aleem, S.J. Hashemifar, H. Akbarzadeh, Physica B 400, 297 (2007).

[14] D. Madouri, M. Ferhat, Phys. Status Solidi B 242, 2859 (2005)

[15] M. Ferhat, A. Zaoui, Appl. Phys. Lett. 88, 161902 (2006).

[16] F. Wooten, Optical Properties of Solids, Academic, New York 1972.

[17] Y. Shen, Z. Zhou, J. Appl. Phys. 103, 074113 (2008).

[18] R. Riane, A. Zaoui, S.F. Matar, A. Abdiche, Physica B 405, 985 (2010). 